\title{
Effect of Tanshinone IIA on the Noncovalent Interaction Between Warfarin and Human Serum Albumin Studied by Electrospray Ionization Mass Spectrometry
}

\author{
Jie Liu, ${ }^{\text {a }}$ Xiaoru Wang, ${ }^{\text {a,c }}$ Zongwei Cai, ${ }^{b}$ and Frank S. C. Lee \\ ${ }^{a}$ Department of Chemistry and The Key Laboratory of Analytical Science of MOE, College of Chemistry and \\ Chemical Engineering, Xiamen University, Xiamen, China \\ ${ }^{\mathrm{b}}$ Department of Chemistry, Hong Kong Baptist University, Kowloon, Hong Kong SAR, China \\ ${ }^{\mathrm{c}}$ Key Lab on Analytical Technology Development and Standardization of Chinese Medicines, First Institute \\ Oceanography of SOA, Qingdao, China
}

Enhanced anticoagulation and/or even bleeding are often observed when patients on long-term warfarin (WAR) therapy consumed Danshen, a well-known medicinal herb in traditional Chinese medicine (TCM). This study demonstrates that altered WAR metabolism, arising from its interaction with the active components in Danshen, played a significant role in this curative effect. Mass spectrometric techniques including ESI-ITMS (electrospray ionization ion-trap mass spectrometry) and ESI-TOF (time-of-flight)-MS have been developed for the study of such drug-herb interactions. The experimental approach involved a detailed analysis and comparison of WAR metabolites in vivo from blood or urine of rats that had been orally administrated with WAR, either singly or together with the representative bioactive component of Danshen-lipid soluble TIIA (Tanshinon IIA), and a study of the interaction of human serum albumin (HSA), WAR, and water-soluble sodium tanshinone IIA sulfonate (STS) in vitro. Results demonstrate that TIIA accelerates the metabolic rate of WAR, whereas STS displaces WAR from the WAR-HSA complex, resulting in an increase of free WAR concentration in blood. It is suggested that the elevated level and enhanced metabolism of WAR is responsible for the over-anticoagulation effect observed. (J Am Soc Mass Spectrom 2008, 19, 1568-1575) (C) 2008 Published by Elsevier Inc. on behalf of American Society for Mass Spectrometry

$\mathrm{T}$ The root of Salvia miltiorrhiza is known in China as Danshen, which is a widely used traditional Chinese medicine (TCM) and officially listed in the Chinese Pharmacopeia. It is mainly used for the treatment of coronary heart diseases, particularly angina pectoris and myocardial infarction [1]. Tanshinone IIA (TIIA) is one of the representative bioactive components in the lipid-soluble fraction of Danshen [2]. Many biological studies have been carried out on this relatively abundant component. A water-soluble derivative of tanshinone, sodium tanshinone IIA sulfonate (STS), has in fact been tested clinically in coronary heart disease patients with positive results on causing coronary vasodilatation and inhibiting coronary artery spasm [3].

Many medicinal herbs and pharmaceutical drugs are therapeutic at one dose and toxic at another. Interactions between herbs and drugs may increase or decrease the pharmacological or toxicological effects of

Address reprint requests to Professor Xiaoru Wang, Xiamen University, College of Chemistry and Chemical Engineering, Department of Chemistry and The Key Laboratory of Analytical Science of MOE, Xiamen 361005, China. E-mail: xrwang2003@yahoo.com.cn either component, and the subject is the focus of this study. Warfarin (WAR), with its known effect of inhibiting vitamin K-dependent coagulation factors, is a common anticoagulant agent that has been introduced into clinical medicine for more than $40 \mathrm{yr}[4,5]$. It has been reported that anticoagulation could be enhanced, sometimes even to the extent of bleeding, when WAR is dosed together with Danshen. Danshen cannot react with WAR directly under physiological conditions, suggesting the lack of true "pharmacy interaction" between Danshen and WAR. However, some type of pharmacokinetic interaction between the two may have played an important role in Danshen's well-documented effects on blood concentration, clearance, and apparent volume of distribution of WAR [6-11].

The serum albumin, as one of the most abundant carrier proteins, can bind to a number of relatively insoluble endogenous compounds such as unesterified fatty acids, bilirubin and bile acids. It also binds a wide variation of drugs and xenobiotics to facilitate their transport throughout the circulatory system [12-18]. WAR, a highly protein-bound drug, binds to the protein through site 1 , which is a preformed binding pocket 
within the core of subdomain IIA of human serum albumin (HSA) [19, 20]. Danshen is also strongly proteinbound and albumin is the major serum protein that binds to Danshen [21]. As the major component of Danshen, TIIA has been reported to bind to HSA, which can be detected by the red shift of the maximum absorption wavelength of TIIA and an enhancement of the absorbance [22]. So it is proposed that Danshen may change the plasma protein binding of WAR by displacing WAR from its binding sites, resulting in overanticoagulant because of the increased blood concentration of WAR [11]. Like most herbal plants, the bioactive components in Danshen are extremely complex. A study of the interactions involved between WAR and the entire Danshen extract is therefore an ineffective way to sort out the mechanistic pathways between the two. In this study, instead, Danshen IIA was chosen as a representative bioactive species of Danshen, and its interaction with WAR was investigated to gain better insight on the mechanisms involved in protein-drug interactions at a molecular level.

There are several analytical techniques that have been used for the analysis of noncovalently bound complex. Xie et al. [23] used a spectroscopic method to investigate the interaction between 2,3-dihydro-4',5,7trihydroxyflavone and HSA. Kreutz et al. [24] presented a novel concept for the identification of RNA binders by ${ }^{19} \mathrm{~F}$ nuclear magnetic resonance (NMR) spectroscopy. X-ray was also used by Kaiser et al. [25] to analyze the complex eukaryotic $20 S$ proteasome with the natural inhibitor TMC-95A. The chief disadvantage of spectroscopic methods is that they cannot provide a direct readout of the stoichiometry of the binding partners in protein-ligand complexes. Although NMR and X-ray crystallography can yield high-resolution structures of biomolecules and complexes from the solution phase and solid phase, respectively, both of these techniques require relatively large quantities of materials and are time consuming. Moreover, NMR is limited to molecules with molecular weight not exceeding $40 \mathrm{kDa}$; furthermore, not all proteins are easy to crystallize for X-ray crystallography detection [26]. Electrospray ionization mass spectrometry (ESI-MS) has recently emerged as an attractive alternative [27-31]. Although unable to provide direct structural data as NMR and X-ray crystallography, ESI-MS does provide important stoichiometry information of the complex formed between two binding partners. Experimentally, the technique also enjoys the merits of high analytical speed and small sample requirement. ESI is a soft ionization technique that allows for the direct analysis of proteins in their biologically active form in the solution. Because of its high sensitivity and moderate resolution at very high $m / z$ (e.g., above $m / z$ 6000), the time-of-flight (TOF) analyzer coupled with ESI ionization (ESI-TOF-MS) provides an ideal system for the detection of macromolecules and large noncovalent complexes [32, 33], and has been widely used for probing noncovalent proteinligand interactions [34-37].
In this study, ESI-ion trap-MS (ESI-ITMS) was used for the detection of metabolites of WAR in vivo to study the effect of TIIA on the metabolism of WAR. Then ESI-TOF-MS was applied to monitor the interactions among Danshen, WAR, and HSA. This approach represents the first such attempt in the literature. The mechanism of interaction between Danshen and WAR and how this interaction affects the noncovalent binding of WAR with HSA are also the main topics of interest.

\section{Experimental}

\section{Material and Reagents}

Warfarin (WAR), tanshinone IIA (TIIA), and sodium tanshinone IIA sulfonate (STS) were purchased from the National Institute for the Control of Pharmaceutical and Biological Products (NICPBP, Beijing, China). Human serum albumin (HSA, fraction V) was purchased from Sigma Chemical Co. (St. Louis, MO, USA).

Ammonium acetate was from Fluka (Zwijndrecht, The Netherlands). HPLC-grade methanol and acetonitrile were purchased from Tedia (Fairfield, OH, USA). Purified deionized water (18.2 M 2 ) prepared by a Milli-Q Ultrapure water system (Millipore, Bedford, MA, USA) was used for all buffers. Male SpragueDawley (S.D.) rats were purchased from Laboratory Animal Services Centre, the Chinese University of Hong Kong. Other reagents used were of analytical grade purity.

\section{Preparation of the Metabolites of TIIA and WAR In Vivo}

Male S.D. rats with weight between 200 and 220 g were under the process of oral administration as described previously [38]. Three rats were sorted to a group. WAR $(4 \mathrm{mg})$ and a mixture of WAR $(4 \mathrm{mg})$ and TIIA $(4 \mathrm{mg})$ were each orally administrated to the rats of different groups. Urine was collected up to $12 \mathrm{~h}$ after oral administration. The samples were kept at $-20{ }^{\circ} \mathrm{C}$ before pretreatment. Then the rats were decapitated and the blood was collected with antithrombin heparin. Based on the method described by Qian [39], blood was first centrifuged at $8200 \times g$ for 20 min to collect the plasma. Then $300 \mu \mathrm{L}$ of plasma sample was measured and put into a $1.5 \mathrm{~mL}$ polystyrene tube with $1 \mathrm{~mL}$ methanol, mixed well, and then centrifuged at $8200 \times g$ for $20 \mathrm{~min}$ to precipitate out the protein from the supernatant containing WAR and its metabolites. The supernatant was transferred into another tube and dried under a stream of nitrogen. The residue was reconstituted with $50 \mu \mathrm{L}$ of methanol and then centrifuged at $8200 \times g$ for $20 \mathrm{~min}$. A volume of $5 \mu \mathrm{L}$ of the supernatant was analyzed by liquid chromatography (LC)/MS. Urine samples were also pretreated following the method previously described [38] before LC/MS analysis. 


\section{Preparation of the Noncovalent Complexes} of HSA and Ligands

The HSA stock solution was prepared by dissolving 25 mg HSA in $1 \mathrm{~mL} 20 \mathrm{mM} \mathrm{NH}_{4}$ Ac buffer (pH 7.4). Stock solutions of WAR and STS were respectively prepared in methanol and $\mathrm{NH}_{4} \mathrm{Ac}$ buffer to a concentration of 1 $\mathrm{mg} / \mathrm{mL}$. For the reaction of ligand binding to HSA, the HSA stock solution was added to $20 \mathrm{mM} \mathrm{NH}_{4}$ Ac buffer (pH 7.4) to make a final concentration of $50 \mu \mathrm{M}$. Ligand was then added to the solution followed by thorough mixing. The solution was incubated at $37^{\circ} \mathrm{C}$, and the HSA-WAR complex was produced from the two after a certain incubation time. The dynamic process of the binding reaction was monitored at different concentration ratios of HSA to ligand and at different incubation times. The STS was titrated and the mass variations of HSA-ligand complexes were observed to study the effect of TIIA on WAR binding HSA.

\section{Mass Spectrometry}

A Bruker Esquire 4000 ion trap mass spectrometer (Bruker-Fransen, Bremen, Germany) was used for the ESI-MS analysis of the metabolites of WAR extracted from the bioassay. The operational parameter settings were as follows: pressure of nebulizer gas, $50 \mathrm{psi}$; ion spray voltage, $4500 \mathrm{~V}$; flow rate, $12 \mathrm{~L} / \mathrm{min}$; temperature of the drying gas, $350{ }^{\circ} \mathrm{C}$. Negativ e-ion mode was used in MS analysis with the mass range scanned from $\mathrm{m} / \mathrm{z}$ 100-600. A collision energy of $0.35 \mathrm{~V}$ was used to acquire the fragment ion spectra of the metabolites. Coupled with liquid chromatography, metabolites could be separated from the complex biomatrix before MS analysis. An HP 1100 liquid chromatograph (Hewlett-Packard, Wilmington, DE, USA), equipped with a Phenomenex C18 reverse-phase column $(150 \times$ $2.0 \mathrm{~mm}, 5 \mu \mathrm{m}$ ), was used for separation. $\mathrm{NH}_{4}$ Ac buffers $(5 \mathrm{mM})$ containing $0.1 \%(\mathrm{vol} / \mathrm{vol})$ acetic acid (A) and methanol (B) were used as the HPLC mobile phase. A gradient elution was set as follows: $5 \%$ B was increased to $50 \%$ from 0 to $30 \mathrm{~min}$, then held for $10 \mathrm{~min}$. The flow rate of the mobile phase was set at $0.4 \mathrm{~mL} / \mathrm{min}$.

Protein-ligand complexes were analyzed with an Agilent G1969A TOF mass spectrometer equipped with an ESI source (Agilent Technologies, Palo Alto, CA, USA) under positive-ion mode. Sample solution was directly infused with mobile phase containing $20 \mathrm{mM}$ $\mathrm{NH}_{4} \mathrm{Ac}$ buffer ( $\mathrm{pH}$ 5.0) at a flow rate of $20 \mu \mathrm{L} / \mathrm{min}$. A mixed standard for tuning solution was used for tuning and mass calibration. This instrument was under optimum conditions with a resolution of about 18,000 at $\mathrm{m} / \mathrm{z}$ 2721 and mass error $<3$ ppm. Relevant parameters were adjusted to obtain optimum resolution for analysis of the protein-ligand complex. The temperature, flow rate of the drying gas, and nebulizer pressure were set at $270{ }^{\circ} \mathrm{C}, 7 \mathrm{~L} / \mathrm{min}$, and $15 \mathrm{psi}$, respectively. The potential of capillary and skimmer were set at 2700 and $70 \mathrm{~V}$. Enhancing the potential of fragmentor and octu- pole radio frequency voltage (Oct RFV) increases the sensitivity for detecting ions with high $\mathrm{m} / \mathrm{z}$. So the fragmentor was set at $400 \mathrm{~V}$ and Oct RFV was set at $300 \mathrm{~V}$. The scanning mass range of the mass spectrometer was from $\mathrm{m} / \mathrm{z} 600$ to 6000 .

\section{Results and Discussion}

\section{Metabolites of TIIA and WAR In Vivo}

WAR was metabolized in the liver by the cytochrome P450 system [40]. Like other extrinsic matters, WAR also undergoes two metabolic phases in vivo. The principal metabolism of WAR in phase I was catalytically converted to a series of monohydroxylated metabolites. Some minor metabolites such as dehydrowarfarin and warfarin alcohols were also formed. In phase II, the hydroxylated warfarin metabolites were mainly glucuronidated and sulfated before being excreted into urine $(\sim 80 \%)$ and feces $(\sim 20 \%)[5,41,42]$. When TIIA was metabolized together with WAR in vivo, no new additional metabolites other than those observed in pure WAR systems as described earlier were found in urine or blood. The observation suggests the absence of metabolic reactions or pharmacy interactions between the two species.

No WAR was detected in the urine sample collected $12 \mathrm{~h}$ after oral administration with WAR (Figure 1aI). However, WAR was found in the urine if the rat was orally administered WAR together with TIIA (Figure 1aII). Besides WAR itself, warfarin alcohol (Figure 1bII), dehydro-warfarin (Figure 1cII), several monohydroxylated metabolites of WAR (Figure 1dII), glucuronidation of hydroxy-warfarin (Figure 1eII) and sulfated conjugate of hydroxy-warfarin (Figure 1fII) were also detected in the urine excreted from rats dosed with the mixture containing WAR and TIIA. However, except for a very low concentration of the sulfated conjugate of hydroxy-warfarin (Figure 1fI), few other metabolites were observed in the urine sample from rats dosed with WAR alone (Figure 1b, c, d, eI). Negative-ion MS/MS spectra of WAR and its metabolites are shown in Figure 2. All metabolites have a structure similar to that of WAR, but individual metabolites differ in characteristic substituents, thus producing their own characteristic MS/MS spectra. Figure $2 \mathrm{~b}$ shows the MS/MS spectrum of warfarin-alcohol. The fragment of $-\mathrm{CH}_{2} \mathrm{CH}(\mathrm{OH}) \mathrm{CH}_{3}$ is lost, indicating the characteristic structure of warfarin-alcohol. Dehydro-warfarin loses a particular fragment of $\mathrm{O}=\mathrm{C}=\mathrm{CH}_{2}$ in the MS/MS process (Figure 2c). Compared with the MS/MS spectrum of WAR (Figure 2a), those of hydroxy-warfarin show the loss of one more $\mathrm{H}_{2} \mathrm{O}$, indicating the presence of an additional hydroxy group in this metabolite. The MS/MS spectra of the two phase II metabolites, glucuronidation of hydroxy-warfarin and the sulfated conjugate of hydroxy-warfarin, are shown in Figure 2e and f, respectively. The two metabolites behave similarly by first 


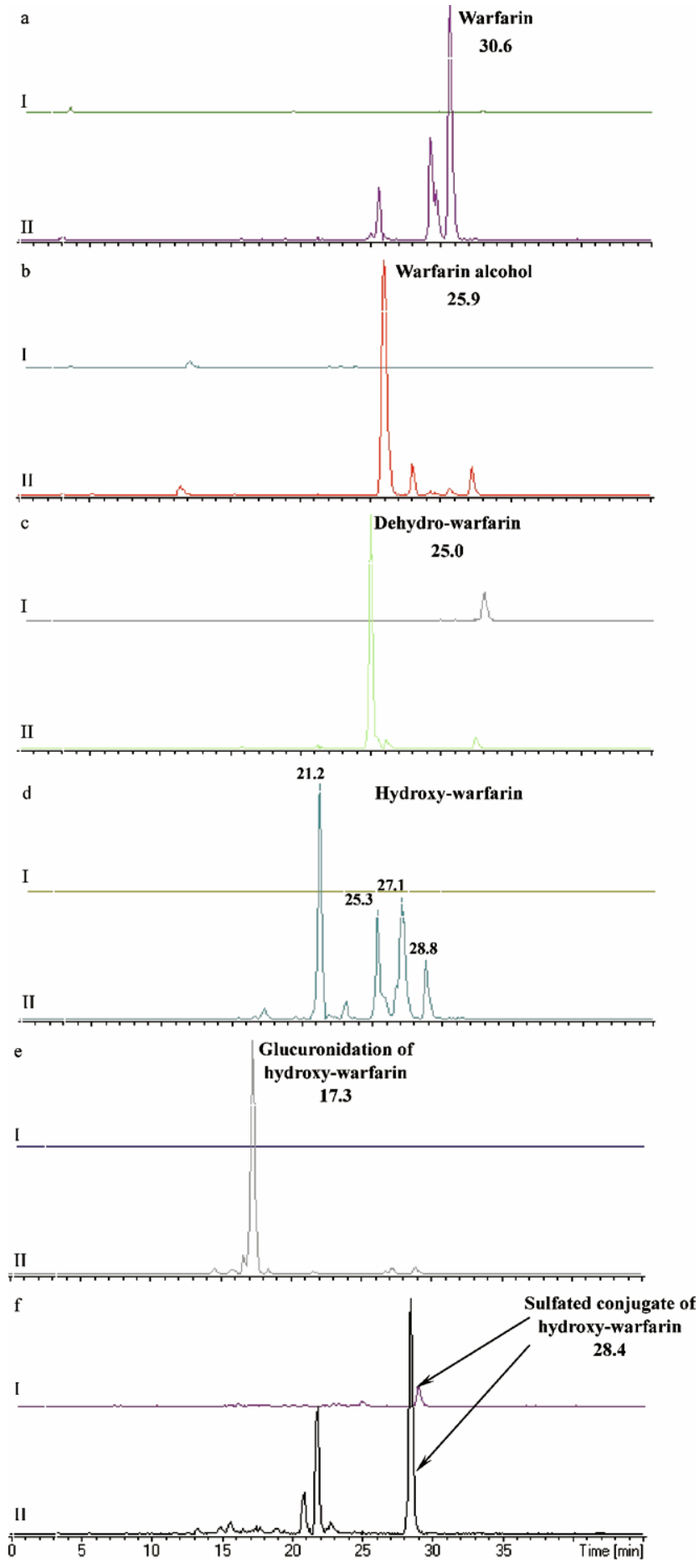

Figure 1. Extracted ion chromatograms of warfarin and its metabolites in urine samples under the in vivo metabolism of warfarin single (I) and with tanshinone IIA (II).

losing their characteristic fragments of glucuronidation and sulfate, respectively, followed by a loss of $\mathrm{CH}_{3} \mathrm{COCH}_{3}$, which is a characteristic fragment in the MS/MS spectrum of WAR (Figure 2a). The results acquired from the blood samples were similar. Metabolites of WAR were clearly detectable in blood from rats dosed with a mixture of WAR and IIA, but few metabolites of WAR were detected if rats were dosed by WAR alone (data not shown). These results show that dosing of the mixture increased the concentration of free WAR in blood. Such an increase could have accelerated the rate of metabolism of WAR, resulting in an increase of the varieties and concentrations of metabolites in urine in a time period of $12 \mathrm{~h}$. In general, the drugs, once entering the organism, will bind first with serum albumin. They are then transported through blood circulation to the receptors and other tissues. At the same time, a dynamic balance exists among the free drug molecules and the bound drugs. The bound drugs would gradually release the drugs in free forms. The free drugs are then metabolized by related enzymes in liver and other organs [43] and result in curative or toxic effects after reactions at the receptor sites. So the mechanism upon the addition of TIIA that accelerated the rate of metabolism of WAR is considered as the replacement of WAR by TIIA at the WAR-HSA binding site. The process increases the concentration of free drugs in blood and, in turn, the metabolic reaction rate of WAR.

\section{Noncovalent Interaction between HSA and WAR}

Similar to other proteins, a typical ESI mass spectrum of the HSA molecules consists of clusters of ion peaks with consecutive multiple charges that originate from the attachment of different number of protons. Figure 3 shows the two clusters of ion peaks in the mass spectrum of HSA under the conditions described earlier in the experimental section. The cluster of ion peaks with high charge states indicates the partially denaturized HSA. Here the HSA molecules have an unfolded configuration, thus making available more space and sites on the molecules for proton binding. In the mass spectrum of the other cluster of ion peaks, four charge states ranging from $15+$ to $19+$ were observed, corresponding to HSA in its native state [44, 45]. At this state, the HSA can bind effectively with ligands to form noncovalent complexes, and such complex formation between WAR and HSA with different protein-ligand ratios has been reported [46, 47].

To study the mechanistics of WAR-HSA interactions, a series of mixtures of WAR and HSA with molar ratios of 1:1, 2:1, 5:1, 10:1, and 20:1 WAR to HSA were prepared in $20 \mathrm{mM}$ ammonium acetate buffer ( $\mathrm{pH} 7.4)$, making a final HSA concentration of $50 \mu \mathrm{M}$. After thorough mixing, they were incubated in a $37^{\circ} \mathrm{C}$ water bath for $2 \mathrm{~h}$. Figure 4a compares the molar ratio of the HSA-WAR complex to HSA as quantified by their relative MS intensity at different WAR/HSA ratios after $2 \mathrm{~h}$ of incubation. When WAR and HSA were incubated with the ratios of $1: 1$ and 2:1, only small amounts of complexes were observed. When the ratio was increased to 5:1, the relative abundance of complex to HSA increased noticeably. A further increase of the WAR to HSA ratio beyond 5:1 resulted in little change 

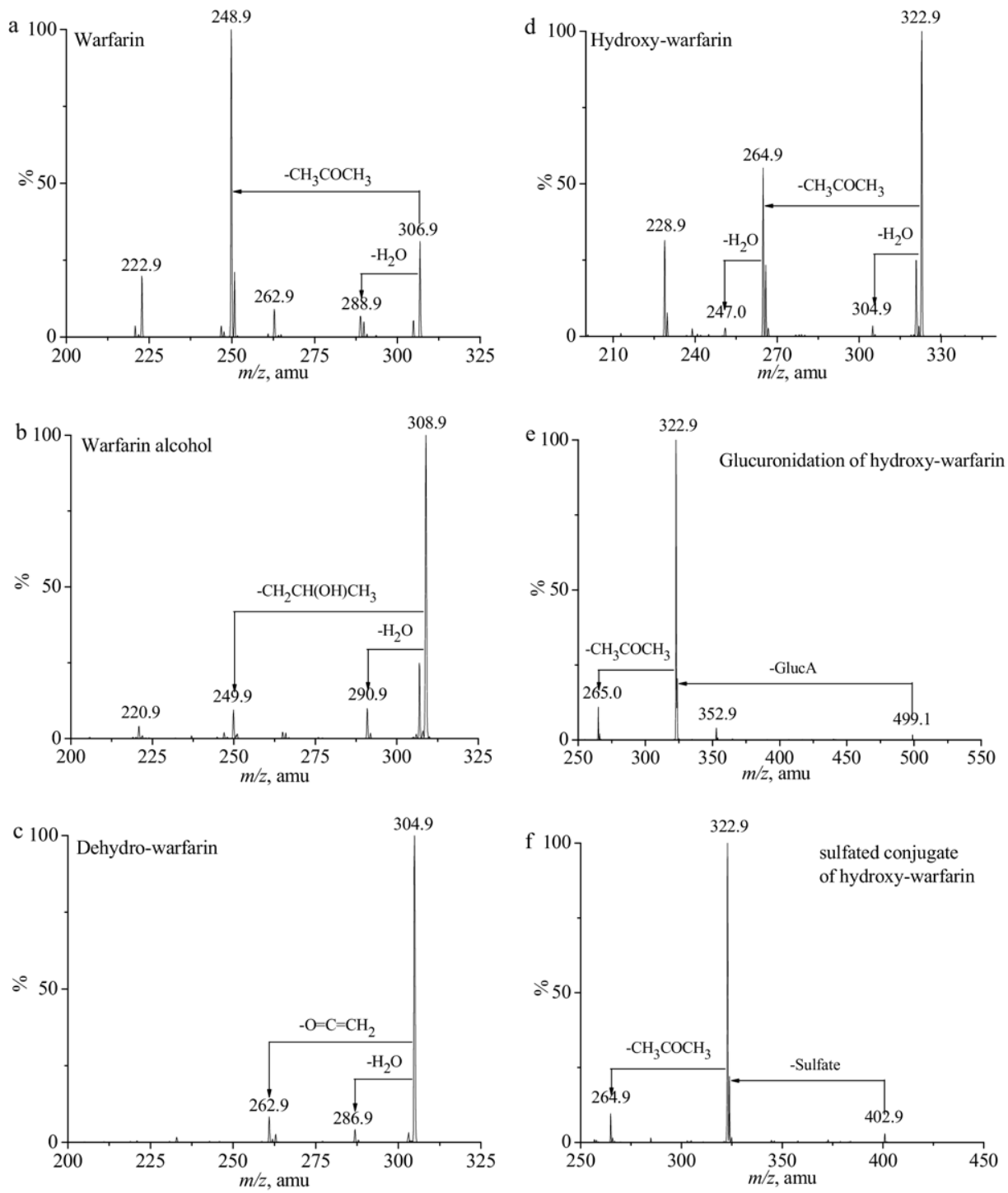

Figure 2. MS/MS spectra of the $[\mathrm{M}-\mathrm{H}]^{-}$ions of warfarin and its metabolites in vivo under negative-ion mode.

of the ratio of complex to HSA. Then different incubation times were studied at a constant WAR to HSA ratio (5:1). The effect of incubation time on the formation of the HSA-WAR complex is shown in Figure 4b. The relative abundance of the complex relative to HSA reached the maximum after $30 \mathrm{~min}$ incubation. In the following $1.5 \mathrm{~h}$, the relative abundance stayed at about the same level. Based on the results, an incubation time of $30 \mathrm{~min}$ and a ratio of WAR to HSA at 5:1 were chosen as the optimum conditions for further experiments on the noncovalent interaction of WAR and HSA in vitro by direct ESI-TOF-MS study as described in the following text.

\section{Reactions among HSA, WAR, and STS}

Figure 5a shows the MS spectrum of a reaction mixture at 1:5 $\mathrm{M}$ ratio of HSA to WAR. At the beginning of incubation, only signals of free HSA were observed, i.e., an intact HSA molecular ion with multiple charge states between $15+$ and $19+$, corresponding to the molecular weight of $66,429 \pm 2 \mathrm{Da}$. After $30 \mathrm{~min}$ incubation at $37^{\circ} \mathrm{C}$, additional peaks corresponding to complex formation between HSA and one WAR ligand with a mass of $66,737 \pm 2$ Da was observed after deconvolution (Figure $5 \mathrm{~b}$ ). Since the molecular weight of WAR is 308, the observed mass thus corresponded to a stoichiometry of 1:1 $\mathrm{M}$ ratio for the complex of HSA to WAR. Then STS with the same concentration as that of WAR was added to the incubation solution, and the interaction between STS and complex HSA-WAR was also monitored at different times. Figure $5 \mathrm{c}-\mathrm{e}$ shows the mass spectra and mass variation of HSA and the complexes after incubation for 0,15 , and $30 \mathrm{~min}$, respectively. Besides the ion peaks of HSA, several additional peaks were 


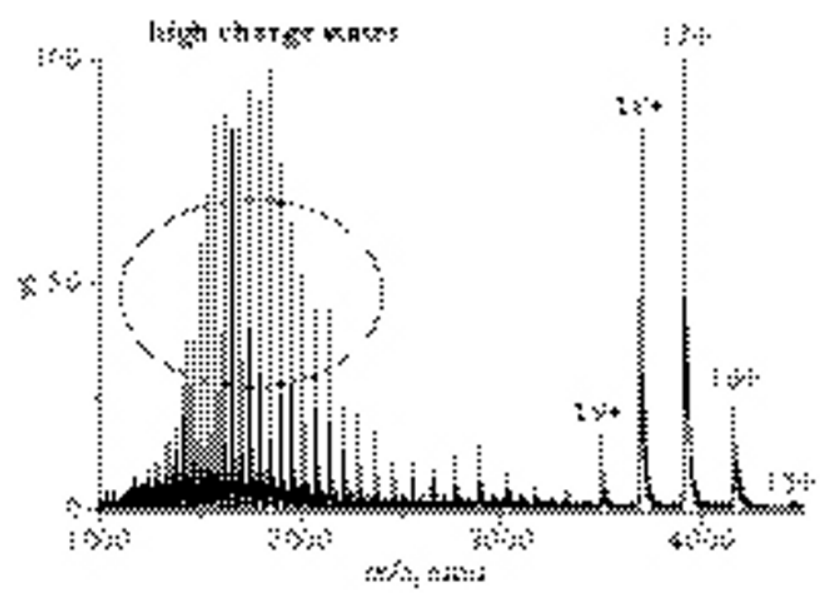

Figure 3. ESI-mass spectrum of HSA with denatured and intact forms.

observed in the incubation process, and these corresponded to HSA-ligand complexes with the same charge states as that of native HSA. For the mass variation of the complex, the relative abundance of HSA-WAR decreased with the increase of a new peak. The new peak with a mass of $66,802 \pm 2 \mathrm{Da}$ represented a mass increase of 373 Da over the parent HSA, corresponding to the mass of STS (396 Da) without a sodium atom. Because STS existed in the form of tanshinone IIA sulfonate (TS) and $\mathrm{Na}^{+}$ions in water, TS will substitute STS to bind with HSA, resulting in the formation of a complex of HSA-TS with a molecular mass of $66,802 \pm 2 \mathrm{Da}$ and a stoichiometry of 1:1 HSA to TS. The result indicated that the addition of STS indeed affected the noncovalent binding between HSA and WAR by competing with WAR for the same binding site on HSA. As a result, more bound WAR molecules were set free, along with an increase of the amount of HSA-TS complex at the expense of those of HSA-WAR.

\section{Conclusions}

An ESI-ITMS-based method was developed for the study of WAR-Danshen interactions. Results demonstrated that the active component of Danshen, TIIA, affects the pharmacokinetics of WAR through an alteration of its metabolism. Monitored by ESI-ITMS, WAR and its metabolites were detected with high concentrations in urine and blood collected from the rats orally dosed by WAR together with TIIA. When WAR alone was orally administrated to rats, a low level of WAR and only a few of its metabolites were detected in urine or blood.

An ESI-TOF-MS-based method was developed for the study of noncovalent interactions of HSA and ligands. A complex of HSA-WAR with the stoichi- ometry of 1:1 was detected when WAR was incubated together with HSA in vitro. STS, a water-soluble derivative of TIIA having the same curative effect as that of TIIA, was able to effectively reduce the relative concentration of HSA-WAR complex while enhancing the relative concentration of a different complex, HSA-TS, indicating the ability of TS to replace WAR from its binding site on the HSA-WAR complex. These results explained the over-anticoagulation phenomenon observed in patients treated by WAR together with Danshen.

Overall, our results show that TIIA interacts pharmacokinetically with WAR through affecting the concentration and metabolic pathways of WAR in blood. The study also illustrates the effectiveness of ESI-MS and TOF-MS methodologies in studying the pharmacological interactions between drugs and proteins. Further experiments to understand the mechanistic basis of the curative effects of tanshinones and how it interacts with the biological receptors are in progress.
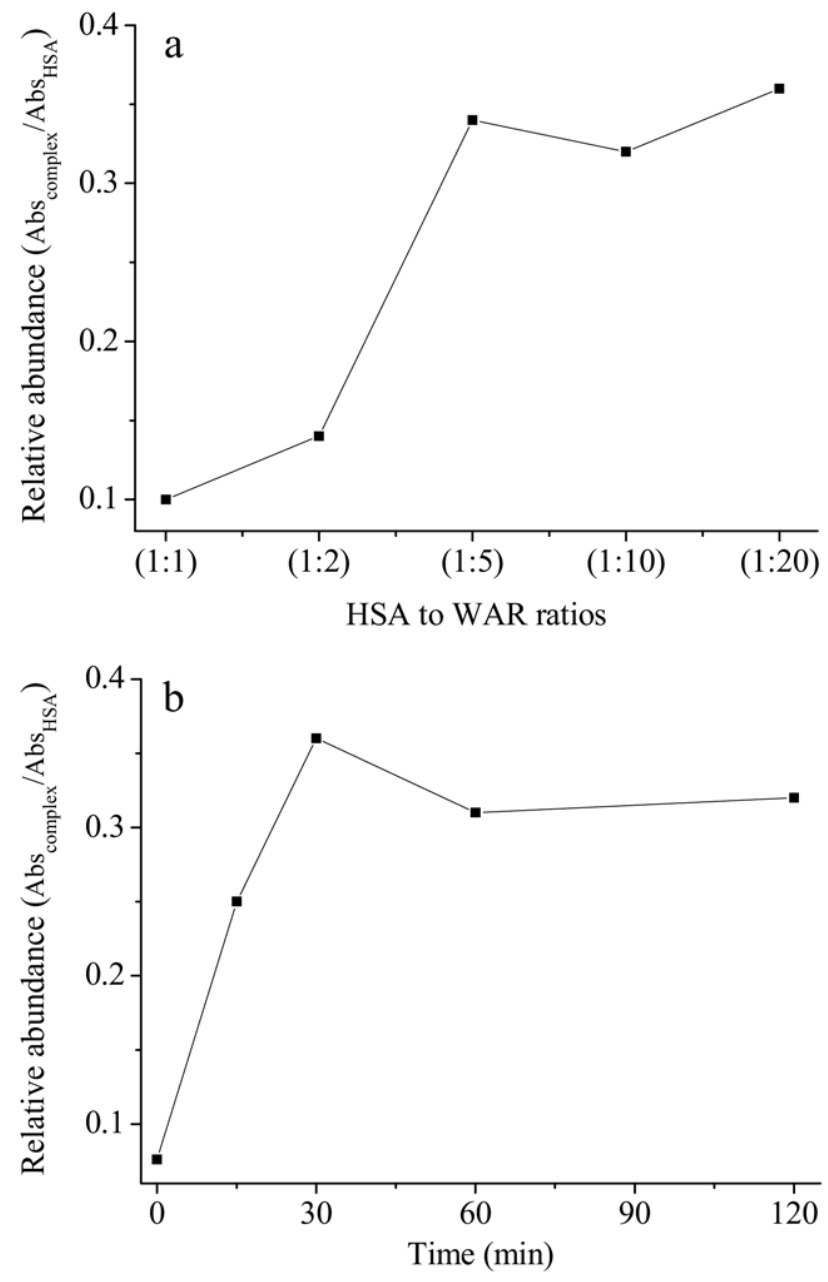

Figure 4. Variations of relative abundance of HSA-WAR complexes formed in reaction mixtures containing different WAR to HSA ratios (a) and under different encubation times (b). 

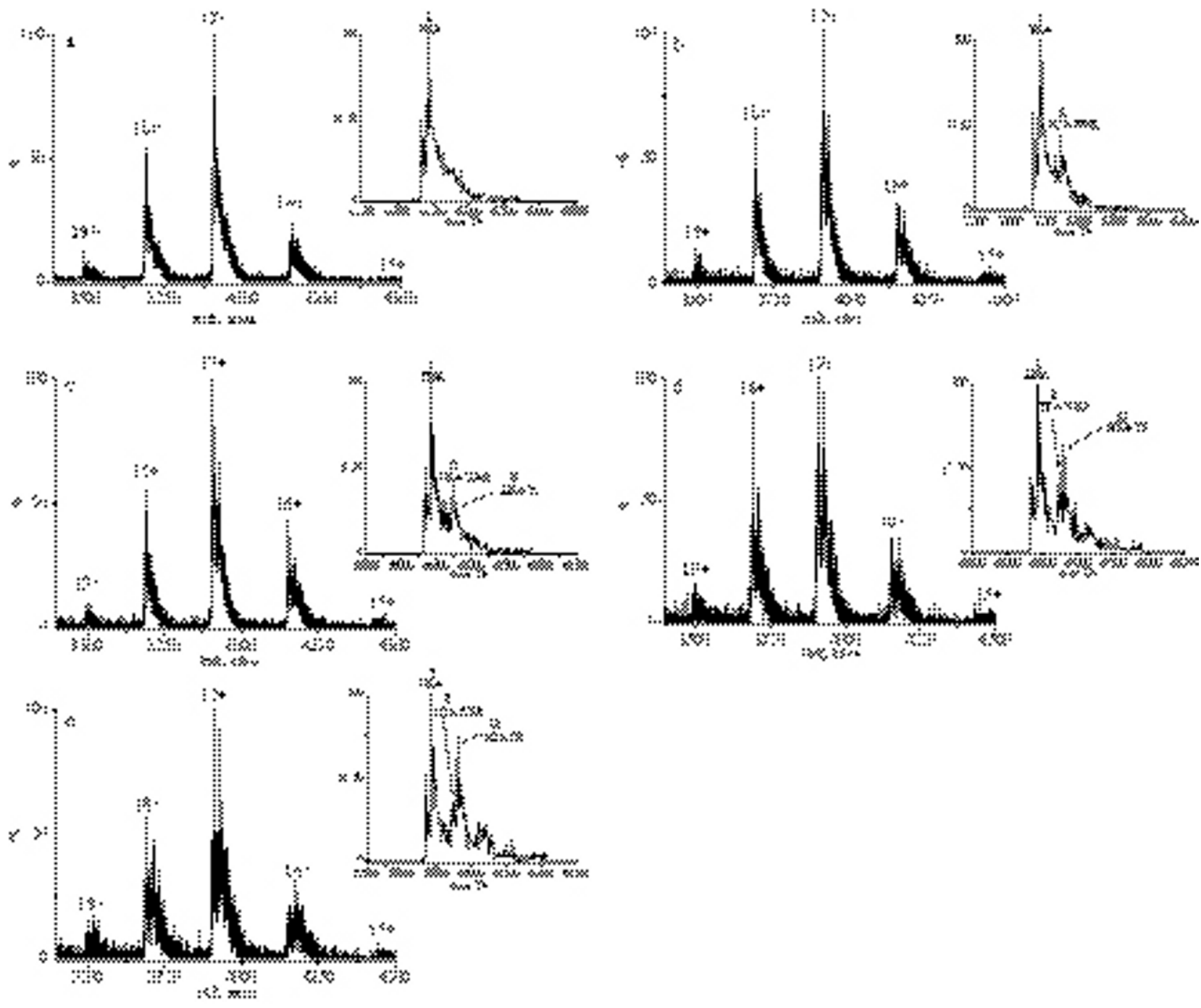

Figure 5. Positive-ion mode ESI mass spectra of a reaction mixture containing HSA and HSA-ligand complexes, as well as their masses after decovolution: (a) Spectrum and mass of HSA obtained at the beginning of addition of WAR. (b) Transformed spectrum and mass of HSA obtained $30 \mathrm{~min}$ after addition of WAR. (c) Spectrum and mass obtained at the beginning of addition of STS into the reaction mixture containing HSA and WAR for 30 min incubation. (d) Transformed spectrum and mass obtained $15 \mathrm{~min}$ after addition of STS. (e) Transformed spectrum and mass obtained $30 \mathrm{~min}$ after addition of STS.

\section{Acknowledgments}

The authors acknowledge financial support for this study from Qingdao Biomedical Research and Testing Center, Laoshan Scientific Department (LS-05-KJZX-76) and the Research Grant Council, University Grants Committee of Hong Kong (HKBU2014/05M).

\section{References}

1. Chinese Pharmacopoeia. Part 1. Chemical Industry Press: Beijing, 2005; pp 52-53.

2. Cai, Z.; Lee, F. S. C.; Wang, X. R.; Yu, W. J. A Capsule Review of Recent Studies on the Application of Mass Spectrometry in the Analysis of Chinese Medicinal Herbs. J. Mass Spectrom. 2002, 37, 1013-1024.

3. Wang, X.; Morris-Natschke, S. L.; Lee, K. H. New Developments in the Chemistry and Biology of the Bioactive Constituents of Tanshen. Med. Res. Rev. 2007, 27, 133-148.

4. Naidong, W.; Ring, P. R.; Midtlien, C.; Jiang, X. Development and Validation of a Sensitive and Robust LC-Tandem MS Method for the Analysis of Warfarin Enantiomers in Human Plasma. J. Pharm. Biomed. Anal. 2001, 25, 219-226.
5. Kaminsky, L. S.; Zhang, Z. Y. Human P450 Metabolism of Warfarin Pharmacol. Ther. 1997, 73, 67-74.

6. Izzo, A. A.; Di Carlo, G.; Borrelli, F.; Ernst, E. Cardiovascular Pharmacotherapy and Herbal Medicines: The Risk of Drug Interaction. Int. J. Cardiol. 2005, 98, 1-14.

7. Zhou, L.; Zuo, Z.; Chow, M. S. S. Danshen: An Overview of Its Chemistry, Pharmacology, Pharmacokinetics, and Clinical Use. J. Clin. Pharmacol. 2005, 45, 1345-1359.

8. Yu, C. M.; Chan, J. C. N.; Sanderson, J. E. Chinese Herbs and Warfarin Potentiation by "Danshen." J. Intern. Med. 1997, 241, 337-339.

9. Fugh-Berman, A. Herb-Drug Interactions. Lancet 2000, 355, 134-138.

10. Heck, A. M.; Dewitt, B. A.; Lukes, A. L. Potential Interactions between Alternative Therapies and Warfarin. Am. J. Health-Syst. Pharm. 2000, 57, 1221-1227.

11. Hu, Z.; Yang, X.; Ho, P. C. L.; Chan, S. Y.; Heng, P. W. S.; Chan, E. Duan, W.; Koh, H. L.; Zhou, S. Herb-Drug Interactions. Drugs 2005, 65 1239-1282.

12. Mahesha, H. G.; Singh, S. A.; Srinivasan, N.; Appu Rao, A. G. A Spectroscopic Study of the Interaction of Isoflavones with Human Serum Albumin. FEBS J. 2006, 273, 451-467.

13. Tang, J.; Luan, F.; Chen, X. Binding Analysis of Glycyrrhetinic Acid to Human Serum Albumin: Fluorescence Spectroscopy, FTIR, and Molecular Modeling. Bioorg. Med. Chem. 2006, 14, 3210-3217. 
14. Li, Y.; He, W.; Liu, J.; Sheng, F.; Hu, Z.; Chen, X. Binding of the Bioactive Component Jatrorrhizine to Human Serum Albumin. Biochim. Biophys. Acta 2005, 1722, 15-21.

15. Qin, C.; Xie, M.; Liu, Y. Characterization of the Myricetin-Human Serum Albumin Complex by Spectroscopic and Molecular Modeling Approaches. Biomacromolecules 2007, 8, 2182-2189.

16. Li, Y.; He, W. Y.; Liu, H.; Yao, X.; Hu, Z. Daidzein Interaction with Human Serum Albumin Studied Using Optical Spectroscopy and Molecular Modeling Methods. J. Mol. Struct. 2007, 831, 144-150.

17. Dufour, C.; Dangles, O. Flavonoid-Serum Albumin Complexation: Determination of Binding Constants and Binding Sites by Fluorescence Spectroscopy. Biochim. Biophys. Acta 2005, 1721, 164-173.

18. Jin, J.; Zhu, J.; Yao, X.; Wu, L. Study on the Binding of Farrerol to Human Serum Albumin. J. Photochem. Photobiol. A: Chemistry 2007, 191, 59-65.

19. Ghuman, J.; Zunszain, P. A.; Petitpas, I.; Bhattacharya, A. A.; Otagiri, M.; Curry, S. Structural Basis of the Drug-Binding Specificity of Human Serum Albumin. J. Mol. Biol. 2005, 353, 38-52.

20. Ascenzi, P.; Bocedi, A.; Notari, S.; Fanali, G.; Fesce, R.; Fasano, M. Allosteric Modulation of Drug Binding to Human Serum Albumin. Mini-Rev. Med. Chem. 2006, 6, 483-489.

21. Gupta, D.; Jalali, M.; Wells, A.; Dasgupta, A. Drug-Herb Interactions: Unexpected Suppression of Free Danshen Concentrations by Salicylate. J. Clin. Lab. Anal. 2002, 16, 290-294.

22. Zhang, L. W.; Yang, P.; Wang, F. Spectroscopic Studies on the Binding of Effective Component of Chinese Herbs with Human Serum Albumin Spectrosc. Spectral. Anal. 2001, 21, 694-696.

23. Xie, M. X.; Xu, X. Y.; Wang, Y. D.; Liu, Y. Spectroscopic Investigation of the Interaction between 2,3-Dihydro-4',5,7-trihydroxyflavone and $\mathrm{Hu}$ man Serum Albumin. Acta Chim. Sinica 2005, 63, 2055-2062.

24. Kreutz, C.; Hahlig, H.; Konrat, R.; Micura, R. A. General Approach for the Identification of Site-Specific RNA Binders by ${ }^{19} \mathrm{~F}$ NMR Spectroscopy: Proof of Concept. Angew. Chem. Int. Ed. 2006, 45, 3450-3453.

25. Kaiser, M.; Groll, M.; Siciliano C.; Assfalg-Machleidt, I.; Weyher, E.; Kohno, J.; Milbradt, A. G.; Renner, C.; Huber, R.; Moroder, L. Binding Mode of TMC-95A Analogues to Eukaryotic 20S Proteasome. ChemBioChem 2004, 5, 1256-1266.

26. Loo, J. A. Studying Noncovalent Protein Complexes by Electrospray Ionization Mass Spectrometry. Mass Spectrom. Rev. 1997, 16, 1-23.

27. Huang, E. C.; Pramanik, B. N.; Tsarbopoulos, A.; Reichert, P.; Ganguly, A. K.; Trotta, P. P.; Nagabhushan, T. L.; Covey, T. R. Application of Electrospray Mass Spectrometry in Probing Protein-Protein and Protein-Ligand Noncovalent Interactions. J. Am. Soc. Mass Spectrom. 1993, 4, 624-630.

28. Bazoti, F. N.; Markides, K. E.; Bergquist, J.; Tsarbopoulos, A. Noncovalent Interaction Between Amyloid- $\beta$-peptide (1-40) and Oleuropein Studied by Electrospray Ionization Mass Spectrometry. J. Am. Soc. Mass Spectrom. 2006, 17, 568-575.

29. Heck, A. J. R.; van den Heuvel, R. H. H. Investigation of Intact Protein Complexes by Mass Spectrometry. Mass Spectrom. Rev. 2004, 23, $368-$ 389.

30. Ashcroft, A. E. Recent Developments in Electrospray Ionisation Mass Spectrometry: Noncovalently Bound Protein Complexes. Nat. Prod. Rep. 2005, 22, 452-464

31. Loo, J. A. Electrospray Ionization Mass Spectrometry: A Technology for Studying Noncovalent Macromolecular Complexes. Int. J. Mass Spectrom. 2000, 200, 175-186.
32. Loo, J. A.; Berhane, B.; Kaddis, C. S.; Wooding, K. M.; Xie, Y.; Kaufman, S. L.; Chernushevich, I. V. Electrospray Ionization Mass Spectrometry and Ion Mobility Analysis of the 20S Proteasome Complex. J. Am. Soc. Mass Spectrom. 2005, 16, 998-1008.

33. McKay, A. R.; Ruotolo, B. T.; Ilag, L. L.; Robinson, C. V. Mass Measurements of Increased Accuracy Resolve Heterogeneous Populations of Intact Ribosomes. J. Am. Chem. Soc. 2006, 128, 11433-11442.

34. Pinkse, M. W. H.; Heck, A. J. R.; Rumpel, K.; Pullen, F. Probing Noncovalent Protein-Ligand Interactions of the cGMP-Dependent Protein Kinase Using Electrospray Ionizaiton Time of Flight Mass Spectrometry. J. Am. Soc. Mass Spectrom. 2004, 15, 1392-1399.

35. Chevreux, G.; Potier, N.; Van Dorsselaer, A.; Bahloul, A.; Houdusse, A. Wells, A.; Sweeney, H. L. Electrospray Ionization Mass Spectrometry Studies of Noncovalent Myosin VI Complexes Reveal a New Specific Calmodulin Binding Site. J. Am. Soc. Mass Spectrom. 2005, 16, 1367-1376.

36. Chung, E. W.; Henriques, D. A.; Renzoni, D.; Morton, C. J.; Mulhern, T. D.; Pitkeathly, M. C.; Ladbury, J. E.; Robinson, C. V. Probing the Nature of Interactions in SH2 Binding Interfaces: Evidence from Electrospray Ionization Mass Spectrometry. Protein Sci. 1999, 8, 1962-1970.

37. Rostom, A. A.; Tame, J. R. H.; Ladbury, J. E.; Robinson, C. V. Specificity and Interactions of the Protein OppA: Partitioning Solvent Binding Effects Using Mass Spectrometry. J. Mol. Biol. 2000, 296, 269-279.

38. Liu, J.; Wu, J.; Wang, X.; Cai, Z. Study of the Phase I and Phase II Metabolism of a Mixture Containing Multiple Tanshinones Using Liquid Chromatography/Tandem Mass Spectrometry. Rapid Commun. Mass Spectrom. 2007, 21, 2992-2998.

39. Qian, T.; Cai, Z.; Wong, R. N. S.; Jing, Z. Liquid Chromatography/Mass Spectrometric Analysis of Rat Samples for In Vivo Metabolism and Pharmacokinetic Studies of Ginsenoside Rh2. Rapid Commun. Mass Spectrom. 2005, 19, 3549-3554.

40. Honkakoski, P.; Arvela, P.; Juvonen, R.; Liang, M. A.; Kairaluoma, M. Pelkonen, O. Human and Mouse Liver Coumarin 7-Hydroxylases Do Not Metabolize Warfarin In Vitro. Br. J. Clin. Pharmacol. 1992, 33, 313-317.

41. Ufer, M. Comparative Pharmacokinetics of Vitamin K Antagonists. Clin. Pharmacokinet. 2005, 44, 1227-1246.

42. Lewis, R. J.; Trager, W. F. Warfarin Metabolism in Man: Identification of Metabolites in Urine. J. Clin. Invest. 1970, 49, 907-913.

43. Yu, B.; Wu, H.; Zhou, P. The Interaction of Dyes, Drugs and Environmental Chemical Pollutants with Serum Albumin. Chemistry 2005, 68, $1-6$.

44. Henrotte, V.; Laurent, S.; Gabelica, V.; Vander Elst, L.; Depauw, E.; Muller, R. N. Investigation of Non-Covalent Interactions between Paramagnetic Complexes and Human Serum Albumin by Electrospray Mass Spectrometry. Rapid Commun. Mass Spectrom. 2004, 18, 1919-1924.

45. Henrotte, V.; Vander Elst, L.; Laurent, S.; Muller, R. N. Comprehensive Investigation of the Non-Covalent Binding of MRI Contrast Agents with Human Serum Albumin. J. Biol. Inorg. Chem. 2007, 12, 929-937.

46. Rich, R. L.; Day, Y. S. N.; Morton, T. A.; Myszka, D. G. High-Resolution and High-Throughput Protocols for Measuring Drug/Human Serum Albumin Interactions Using BIACORE. Anal. Biochem. 2001, 296, 197207

47. Benkestock, K.; Edlund, P.; Roeraade, J. Electrospray Ionization Mass Spectrometry as a Tool for Determination of Drug Binding Sites to Human Serum Albumin by Noncovalent Interaction. Rapid Commun. Mass Spectrom. 2005, 19, 1637-1643. 